\title{
WEAK CONTINUITY OF BANACH ALGEBRA PRODUCTS
}

\author{
BY CARLOS E. KENIG AND HORACIO PORTA ${ }^{1}$ \\ Communicated by Robert Bartle, January 14, 1975
}

1. This note is based on the remark that a variety of common Banach algebras are dual Banach spaces. For example, a well-known result of Sakai (see [6]) states that von Neumann algebras are characterized, among the $C^{*}$. algebras, by this property (see $\S 3$ for further examples).

Clearly, in a dual Banach space two additional topologies are ubiquitous companions to the norm: the weak* and the bounded weak* topologies (see [1]). Below we announce several theorems about continuity of the product in these topologies. We thereby obtain known as well as (apparently) new results in concrete instances considered by Pym, Rubel, Shields, Ryff, Shapiro, Conway, Dixmier, et al.

Generally we follow [2] for notations and definitions, with the main exception that we will consider a Banach space $A$ whose dual $A^{*}$ will be denoted by $B$; $a$ will be a typical element of $A$ and $b$ and $c$ will be typical elements of $B$. Also, the bounded weak* topology (see [1]) on $B$ will be denoted by $l w^{*}$ (rather than $b w^{*}$ ) in view of the general setting of [3].

2. Let $X, Y$ and $Z$ be linear topological spaces and let $f: X \times Y \rightarrow Z$ be a bilinear map. Consider the following properties of $f$ :

$\left[\mathrm{J},\left(x_{0}, y_{0}\right)\right]: f(x, y) \rightarrow f\left(x_{0}, y_{0}\right)$ as $x \rightarrow x_{0}, y \rightarrow y_{0}$;

$[\mathrm{J}]: f$ satisfies $\left[\mathrm{J},\left(x_{0}, y_{0}\right)\right]$ for all $\left(x_{0}, y_{0}\right)$;

[L]: $f\left(x, y_{0}\right) \rightarrow f\left(x_{0}, y_{0}\right)$ as $x \rightarrow x_{0}$ for each $y_{0}$;

[R]: $f\left(x_{0}, y\right) \rightarrow f\left(x_{0}, y_{0}\right)$ as $y \rightarrow y_{0}$ for each $x_{0}$;

then

$$
[\mathrm{J},(0,0)]+[\mathrm{L}]+[\mathrm{R}] \Longleftrightarrow[\mathrm{J}]
$$

and none of the three properties in the left-hand term is redundant in general.

We assume from now on that there is a norm continuous bilinear map

AMS (MOS) subject classifications (1970). Primary 46B99, 46H99; Secondary 46J15, 46L10, 46L20, 47B05.

1 This first author is a Victor J. Andrew Fellow; second author supported by NSF Grants. 
$B \times A \rightarrow A$ which will be denoted by $(a, b) \rightarrow b \vdash a$. We dualize $\vdash$ to obtain a bilinear norm continuous map $B \times B \rightarrow B$ denoted $(b, c) \longrightarrow b c$ and defined by $\langle a, b c\rangle=\langle b \vdash a, c\rangle$.

Consider for each $a_{0} \in A$, the operator $B \rightarrow A$ given by $b \rightarrow b \vdash a_{0}$ and the following properties for them:

( $\left.\mathrm{w}^{*}-\mathrm{w}\right) w^{*}-w$ continuity; (r) the range of the transpose of $b \rightarrow b \vdash a_{0}$ is contained in $A$; $\left(\ell w^{*}-\mathrm{n}\right) \ell w^{*}$-norm continuity; (k) compact; (f) finite rank; (wk) weakly compact.

In the next theorem the properties in [ ] refer to the product associated to 1 .

2.1 THEOREM. For the $w^{*}$-topology

(i) [R] always holds,

$$
\begin{aligned}
(f) & \Leftrightarrow[J,(0,0)] \Leftrightarrow[J] \Leftrightarrow(f)+[L] \Leftrightarrow(f)+\left(w^{*}-w\right) \\
& \Rightarrow[L] \Leftrightarrow(r) \Leftrightarrow\left(w^{*}-w\right),
\end{aligned}
$$

and the single implications cannot be reversed in general,

(iii) [L] holds for each if and only if $A$ is reflexive.

2.2 THEOREM. For the $\ell w^{*}$-topology

(i) $(\mathrm{k}) \Leftrightarrow[\mathrm{J},(0,0)]$,

$$
\begin{aligned}
{[\mathrm{J}] } & \Leftrightarrow\left[\mathrm{J},\left(0, c_{0}\right)\right] \Leftrightarrow[\mathrm{J},(0,0)]+[\mathrm{L}] \\
& \Leftrightarrow[\mathrm{L}]+(\mathrm{k}) \Leftrightarrow\left(\ell \mathrm{w}^{*}-\mathrm{n}\right),
\end{aligned}
$$

(iii) $[\mathrm{L}] \Rightarrow$ (wk) and the implication cannot be reversed in general.

3. EXAmple 1. Let $G$ be a locally compact group, $A=C_{0}(G)$ the space of continuous functions vanishing at infinity under the sup norm. Then $B$ is the space of finite Radon measures on $G$. For $f \in A, \mu \in B$, take $\mu \vdash f$ to be the function

$$
(\mu \vdash f)(s)=\int_{G} f(t s) d \mu(t) .
$$

The product associated to $\vdash$ is the convolution of finite measures.

EXAmple 2. Let $\Omega$ be a connected open set of the complex plane supporting nonconstant bounded holomorphic functions (e.g., a disc). Let $B=$ $H^{\infty}(\Omega)$ be the space of bounded holomorphic functions on $\Omega$ under the sup norm. Then (see [5]) $B=H^{\infty}(\Omega)$ is the dual of $A=L^{1}(\Omega) / N$ where 


$$
N=\left\{f \in L^{1}(\Omega) ; \iint f h d x d y=0 \text { for each } h \in H^{\infty}(\Omega)\right\} \text {. }
$$

Define $h \vdash(f+N)=h f+N$. The product associated to this $\vdash$ is the ordinary function product. See [5] for a detailed study of this space.

ExAmple 3. Let $H$ be a Hilbert space, $B=L(H)$ the space of all bounded linear operators in $H . B$ is the dual of $A=L_{*}(H)$, the trace class (see [6]). Then, $T \vdash S=S T$ for $T \in L(H), S \in L_{*}(H)$ induces the operator product in $L(H)$.

EXAMPLE 4. Let $A=\ell_{q}(S), B=\ell_{p}(S)$ with $1 / p+1 / q=1, p, q>1$, and $p=\infty$ for $q=1$. We can consider an arbitrary $\vdash$ product and the pointwise product. The main feature needed here is that all operators $\ell_{p} \rightarrow \ell_{q}$, $2<p \leqslant \infty$, are compact (see [4]).

Example 5. $A=c_{0}(S), B=\ell_{1}(S)$ with pointwise product.

EXAmple 6. Let $(X, \Sigma, \mu)$ be a finite measure space, $A=L^{1}(X, \Sigma, \mu)$ and $B=L^{\infty}(X, \Sigma, \mu)$. The product here is again the pointwise product.

Then

\begin{tabular}{|c|c|c|}
\hline Ex & {$[\mathrm{J}]$ for $w^{*}$} & {$[\mathrm{~J}]$ for $\ell w^{*}$} \\
\hline 1 & holds iff $G$ is finite & holds iff $G$ is compact \\
\hline 2 & never holds & always holds \\
\hline 3 & holds iff $\operatorname{dim} H<\infty$ & holds iff $\operatorname{dim} H<\infty$ \\
\hline 4 & $\begin{array}{l}\text { holds for pointwise } \\
\text { product iff } S \text { is } \\
\text { finite }\end{array}$ & $\begin{array}{l}\text { holds for all } \vdash \text {-products } \\
\text { if } 2<p \leqslant \infty \text {; holds for } \\
\text { pointwise product if } \\
1<p \leqslant \infty\end{array}$ \\
\hline 5 & $\begin{array}{l}\text { holds iff } S \text { is } \\
\text { finite }\end{array}$ & always holds \\
\hline 6 & holds iff $\Sigma$ is finite & holds iff $\mu$ is purely atomic \\
\hline
\end{tabular}

\section{REFERENCES}

1. M. M. Day, Normed linear spaces, 3rd ed., Springer-Verlag, New York, 1972.

2. N. Dunford and J. T. Schwartz, Linear operators. I: General theory, Pure and Appl. Math., vol. 7, Interscience, New York, 1958. MR 22 \#8302.

3. H. Porta, Compactly determined locally convex topologies, Math. Ann. 196 (1972), 91-100. MR 45 \#5705. 
4. H. P. Rosenthal, On quasi-complemented subspaces of Banach spaces, with an appendix on compactness of operators from $L^{p}(\mu)$ to $L^{r}(\nu)$, J. Functional Analysis 4 (1969), 176-214. MR 40 \#3277.

5. L. A. Rubel, Bounded convergence of analytic functions, Bull. Amer. Math. Soc. 77 (1971), 13-24. MR 42 \#5025.

6. S. Sakai, $W^{*}$-algebras, Springer-Verlag, Berlin and New York, 1970.

DEPARTMENT OF MATHEMATICS, UNIVERSITY OF CHICAGO, CHICAGO, ILLINOIS 60637

DEPARTMENT OF MATHEMATICS, UNIVERSITY OF ILLINOIS, URBANA, ILLINOIS 61801 